\title{
El sistema nacional de garantías PYME y su contribución al crecimiento económico en México 2003-2008
}

The national small and medium enterprise guarantees and its contribution to economic growth in Mexico 2003-2008

\author{
Alberto Quintal Palomo* \\ Edgar Alfonso Sansores Guerrero** \\ Juana Edith Navarrete Marneou*** \\ * Profesor de la Facultad de Economía \\ de la Universidad Autónoma de Yucatán \\ **Profesores de la Universidad de Quintana Roo \\ qpalomo@uady.mx \\ edsan@uqroo.mx \\ emarneou@uqroo.mx
}




\title{
RESUMEN
}

Actualmente las micro, pequeñas y medianas empresas (PYMES) representan un motor para el crecimiento económico. En México uno de los principales objetivos de las políticas de apoyo a las PYMES es lograr que este sector tenga acceso al financiamiento, por tal motivo se ha implementado un Sistema Nacional de Garantías. El objetivo principal de esta investigación es determinar el impacto del Sistema Nacional de Garantías en el crecimiento económico durante el período 2002-2008.

Palabras claves: Garantias, PYME, racionamiento crediticio, selección adversa.

\section{Clasificación JEL: G38}

\begin{abstract}
In recent times, small business sector as a potencial engine of economic growth. Encouraging lending to small business is the objective of government policy to promote small business, for such a motive government has been implemented system of garantees lending. The purpose of this paper is to determinate the effects of system of garantees lending in the economic growth.
\end{abstract}

Keywords: Garantees lending, small business, credit rationing, adverse select.

JEL Clasification: G38

Revista de Economía, vol. XXVII, núm. 74, primer semestre de 2010. 


\section{I.- Introducción}

En los últimos diez años los programas de apoyo a las micro, pequeñas y medianas empresas (PYMES) representan una prioridad dentro de la política económica en México. De acuerdo con el Instituto Nacional de Estadística, Geografía e Informática (INEGI), ${ }^{1}$ existen dos millones ochocientos veinte mil PYMES, mismas que generan 40 por ciento del Producto Interno Bruto y 64 por ciento del empleo en el país.

A pesar de la implementación de estos programas, las PYMES presentan algunos problemas que inhiben su desarrollo tales como escasa formación de desarrollo de las habilidades empresariales, poca capacidad de producción, insuficientes sistemas de información, desconocimiento del mercado, problemas de comercialización, falta de vinculación con programas para el desarrollo e innovación tecnológica y, en general, con un difícil acceso a esquemas de financiamiento ofrecidos por la banca comercial.

La principal razón por la que las PYMES no crecen y se consolidan, es por la dificultad para acceder a créditos bancarios. De acuerdo con cifras del Banco de México (Banxico), ${ }^{2}$ la banca comercial disminuyó su participación en el PIB de 39.5\% al 16.7\%, esto durante el periodo 1994-2008. Esta disminución fue provocada por la crisis de 1995 y el establecimiento de una política de protección contra el riesgo de no pago por parte de la banca, propiciando un racionamiento del crédito.

En respuesta a la problemática descrita anteriormente, el gobierno de México ha intervenido a través del diseño e implementación de un Sistema Nacional de Garantías cuyo principal proposito es contribuir al crecimiento económico. Esta investigación tiene por objetivo determinar el impacto del Sistema Nacional de Garantías en el crecimiento económico de México durante el período 2002-2008.

\section{II.- La intervención del gobierno en el mercado crediticio}

La justificación de la intervención del gobierno a través de un sistema nacional de garantías se sustenta como una solución para superar el racionamiento del crédito al que se encuentran sometidas las PYME.

\footnotetext{
${ }^{1}$ Censo económico de 2004.

${ }^{2}$ Datos económicos de coyuntura a febrero de 2008.
} 
Sin embargo, resulta transcendental evaluar la pertinencia de dicho programa. Desde la perspectiva económica, el subsidio a un sector puede causar perjuicio a otros, que estarían financiando al primero a través de un subsidio cruzado (Bebczuk, 2000). Esta transferencia intersectorial no mejora la situación económica general y provoca descontento en los sectores afectados.

La teoría económica es categórica en cuanto a la que la única justificación para la intervención del gobierno es la existencia de fallos en el mercado. En el caso del mercado de crédito, los problemas de información asimétrica entran dentro de esa categoría. El grado de imperfección de la información en el mercado del crédito es muy elevado, existiendo costos importantes para los bancos en la captación de la misma (Parker, 2003). En este contexto, la asimetría de información que existe entre acreedores y prestatarios determina que aquellos no dispongan ex-ante de información perfecta sobre las características de los prestatarios, ni puedan anticipar si les beneficiaran las decisiones que los agentes adopten hasta el vencimiento de la deuda, lo que dificulta la evaluación por parte de los acreedores del riesgo de insolvencia presente y futuro de los prestatarios; de hecho, aquellos caracterizados por una mayor probabilidad de insolvencia no tienen incentivos para revelar su nivel de riesgo. El exceso de demanda de crédito a que da lugar este tipo de situaciones puede ser habitual en los mercados crediticios, explicando así la negación de préstamos, la limitación del monto y la exigencia de garantías.

\section{II.a.- Selección adversa}

El efecto de la selección adversa se refiere a un incremento del riesgo medio de la cartera de demandantes inducido por un tipo de interés o en las garantías exigidas por el prestamista (Jaffe y Stiglitz, 1990). Estos altos intereses o garantías desincentivan la solicitud de crédito de los demandantes más adversos al riesgo, originando que los solicitantes tiendan a presentar proyectos con un riesgo más elevado. Si este aumento del riesgo reduce los beneficios esperados por el acreedor, éste no concederá préstamos en esas condiciones, y por tanto, no atenderá la demanda de los agentes sobre los que no pueda estimar si es aceptable el nivel de riesgo de insolvencia. Surge así un racionamiento del crédito en el que no se limita 
el monto de cada potencial demandante sino que se restringe el número de créditos concedidos.

La selección adversa se presenta cuando las entidades bancarias están conscientes de que partiendo de un tipo de interés bajo proceden a elevarlo progresivamente algunos clientes considerarán que el costo crediticio aconseja no llevar a cabo los proyectos de inversión en cartera, decidiendo retirarse del mercado. Estos clientes adversos al riesgo serán los más prudentes ya que, conocedores de que no pueden devolver los créditos que solicitan, serían los primeros en retirarse del mercado, es decir, que al tener que financiar proyectos de menor riesgo y, por tanto, de una menor rentabilidad esperada, los clientes adversos desistirán de su demanda de crédito conforme aumenta su costo.

$\mathrm{El}$ incremento de la tasa de interés por las entidades bancarias disminuirá la calidad media de los clientes ya que la proporción de clientes propensos al riesgo aumentaría, elevándose así el riesgo medio de la cartera de crédito y disminuyendo la probabilidad de éxito de la operación crediticia.

Se denomina selección adversa porque el cliente que está dispuesto a tomar un crédito a cualquier precio se autorrevela ante el banco como un cliente elevado de riesgo. En este caso es la tasa de interés la variable que recoge la información relativa al riesgo del propio contrato de préstamo. Cuando se produce este efecto, el equilibrio no puede obtenerse mediante el análisis de la oferta y la demanda.

\section{II.b.- Riesgo moral}

El riesgo moral es otro efecto derivado de la asimetría de información que, conjuntamente con el efecto de selección adversa, origina racionamiento del crédito. El problema del riesgo moral surge cuando los agentes no soportan plenamente las consecuencias de sus propias acciones y es característico del mercado de seguros, aunque puede aparecer también en el mercado crediticio en determinadas ocasiones. Así, si un prestatario modifica las características, en términos de riesgo, del proyecto e incluso el valor de los flujos de efectivo que observa el banco, podría aparecer este problema.

El problema del riesgo moral puede también aparecer después de realizado el contrato, cuando los prestatarios llevan a cabo acciones no 
observables, o al menos no controlables por el prestamista, que actúan sobre el rendimiento esperado de sus proyectos de inversión, incidiendo negativamente sobre el beneficio esperado por el acreedor. De ello se deriva que el problema relativo a la información disponible para el prestamista se refiere no sólo al desconocimiento del riesgo del cliente sino también a cuál será el riesgo de insolvencia durante el periodo en el que se amortiza la deuda.

Cuando las tasas de interés son elevadas se estimulan los comportamientos oportunistas, ya que los prestatarios tienen incentivos para realizar inversiones más arriesgadas que les permitan obtener rendimientos lo suficientemente elevados como para afrontar el mayor volumen de intereses, pudiendo incluso (en aquellos casos en los que la probabilidad de insolvencia sea muy elevada) abandonar la custodia de los activos aportados como garantía. Por este motivo, el prestamista preferirá denegar el crédito a los prestatarios sujetos a mayores asimetrías informativas, especialmente en un contexto de elevados tipos de interés, para evitar las consecuencias negativas para él del riesgo moral.

La presencia de riesgo moral alterará la relación contractual dado que el prestamista sabe ex-ante que la empresa adoptará la decisión óptima para ella. Por tanto, se verá obligado a dudar de la veracidad de la información relativa a la distribución de flujos de efectivo, ofertando generalmente un contrato por un importe menor al solicitado por la empresa.

\section{III.- Sistemas de garantías de los préstamos}

La presencia de información asimétrica entre acreedores y prestamistas lleva a un equilibrio en donde algunos prestamistas son excluidos. En respuesta a este racionamiento, los gobiernos intervienen en el mercado crediticio a través de subsidios, préstamos directos, capital de riesgo, incentivos fiscales y fondos de garantía crediticia. ${ }^{3}$

En el caso de préstamos directos, una agencia gubernamental actúa como intermediario en lugar del banco; dicha agencia coloca en forma directa créditos a empresas racionadas, obteniendo fondos del mercado de capitales mediante colocación de títulos de deuda o vía impuestos. Los

\footnotetext{
${ }^{3}$ El mecanismo más utilizado y el mejor desarrollado.
} 
préstamos están orientados al sector primario. Los fondos de garantía ${ }^{4}$ permiten a las empresas racionadas el contar con una serie de respaldos tangibles, que en caso de suspensión de pago aseguran la recuperación total o parcial de la deuda a los prestamistas.

Bajo este esquema de garantías, el gobierno se hace responsable del pago de una parte del crédito otorgado a las empresas, las cuales son excluidas por los bancos debido a la carencia de información financiera que compruebe su capacidad de pago. Los bancos administran las garantías y las aplican a los proyectos aprobados o sugeridos por el gobierno. Una vez aprobados los proyectos, si éstos tienen éxito, el banco recibe al pago del capital más los intereses. En caso de fracasar el proyecto, el banco sólo absorbe una parte de la pérdida y el resto es asumida por el gobierno.

Los sistemas de garantía favorecen el acceso a financiamiento a las PYMES, facilitando una garantía parcial o total de la devolución del principal e intereses del prestamista; asumiendo así parte del riesgo en que incurren las instituciones financieras en su actividad crediticia.

Por otra parte, un sistema nacional de garantías debe fomentar el incremento a la inversión productiva. Sin embargo, para las empresas donde la propiedad y la administración no se diferencian entre sí ( como sucede con las PYMES), las decisiones de inversión y consumo no son independientes entre sí, ya que el consumo personal es financiado a través de las utilidades de la empresa. Los individuos prefieran mantener un consumo constante en el tiempo en contrasentido a cambio de un periodo a otro. Como el acceso a deuda más barata aumenta el ingreso futuro, el empresario reparte ese beneficio entre dos periodos, elevando el consumo presente y distorsionando el objetivo del sistema de garantías (Bebczuk, 2001).

\section{IV.- Sistema nacional de garantías 2001-20o8}

En México, la crisis financiera de los años noventa dejó un sistema financiero con enormes carteras vencidas y situaciones de fuerte descapitalización. Esto propició que las instituciones financieras dejaran de otorgar créditos a las empresas que no contaban con: adecuado historial crediticio

\footnotetext{
${ }^{4}$ También conocidos como garantías.
} 
(buró de crédito favorable); utilidades en el negocio; bienes que garantizasen su crédito; algún aval que respondiera por ellos, entre otros múltiples requisitos.

Esta situación originó que las micro y pequeñas empresas fueran percibidas por las instituciones financieras como agentes de alto riesgo, dado que en su mayoría no contaban con planes de negocios, estimaciones adecuadas de crecimiento, suficiente infraestructura, fortalezas patrimoniales, además de carecer en algunos casos de formalidad. Por tal motivo el sistema bancario no las ha considerado como sujetos de crédito, ya que el riesgo percibido y el costo operativo motivaba a la no acreditación y al incremento en la tasa de interés.

Como respuesta a esta problemática, el Gobierno Federal constituyó la Subsecretaría para la Pequeña y Mediana Empresa (SPYME) en el año de 2001, con el fin de coordinar los esfuerzos nacionales para fortalecer las acciones de apoyo contempladas en "El Programa Nacional de Desarrollo 2001-2006”, el cual planteó entre sus principales objetivos el lograr que las micro, pequeñas y medianas empresas obtengan acceso al financiamiento.

En 2001, a través del Fondo de Fomento para la Integración de Cadenas Productivas (FIDECAP) y el Fondo de Apoyo a la Micro, Pequeña y Mediana Empresa (FAMPYME), la Secretaría de Economía aportó 98 millones de pesos a 23 entidades federativas para la constitución o fortalecimiento de programas de garantía constituidos por los Gobiernos de los Estados, no existiendo relación directa entre el operador del fondo y los intermediaros financieros. La banca comercial, al trabajar con estos fondos, se vió obligada a reservar lo mismo que si prestara sin garantías, por lo que no existió un incentivo real para otorgar créditos con garantías de estos fondos.

En 2002 se creó el Fondo de Apoyo para el Acceso al Financiamiento (FOAFI), que tiene como objetivo fomentar y constituir esquemas e instrumentos financieros que faciliten el acceso al crédito y financiamiento a las PYMES, así como incrementar su capacidad de autogestión e impulsar la igualdad de condiciones y oportunidades. Para la implementación de este fondo se requería cumplir con dos aspectos: por una parte era necesario crear las condiciones para que los bancos conocieran el segmento de las Pyme, y eliminar (minimizar) la percepción de riesgo que se tenía de 
ellas: para entender al segmento Pyme como un negocio; por otra parte, se imponía la necesidad de desarrollar la capacidad de gestión de las empresas; esto es, hacer sujetos de crédito a las empresas, todo esto buscando optimizar los recursos del FOAFI.

En 2002 se constituyó el Sistema Nacional de Garantías el cual debía evolucionar hacia un Sistema Nacional de Financiamiento a Micro, Pequeñas y Medianas Empresas (PYMES). El objetivo final es llegar a empresas de menor tamaño, en las mejores condiciones de acceso, con una asistencia adecuada y, principalmente, por medio de productos financieros adecuados a las PYMES. La conformación del Fideicomiso PYME Unicrese $^{5}$ constituyó uno de los principales avances de integración por parte de los intermediarios financieros especializados en reactivar el crédito hacia las PYMES en condiciones más accesibles, tal y como lo muestra el gráfico 1.

\section{Gráfico 1.- Evolución del Crédito Bancario a Empresas y Personas Físicas con Actividad Empresarial}

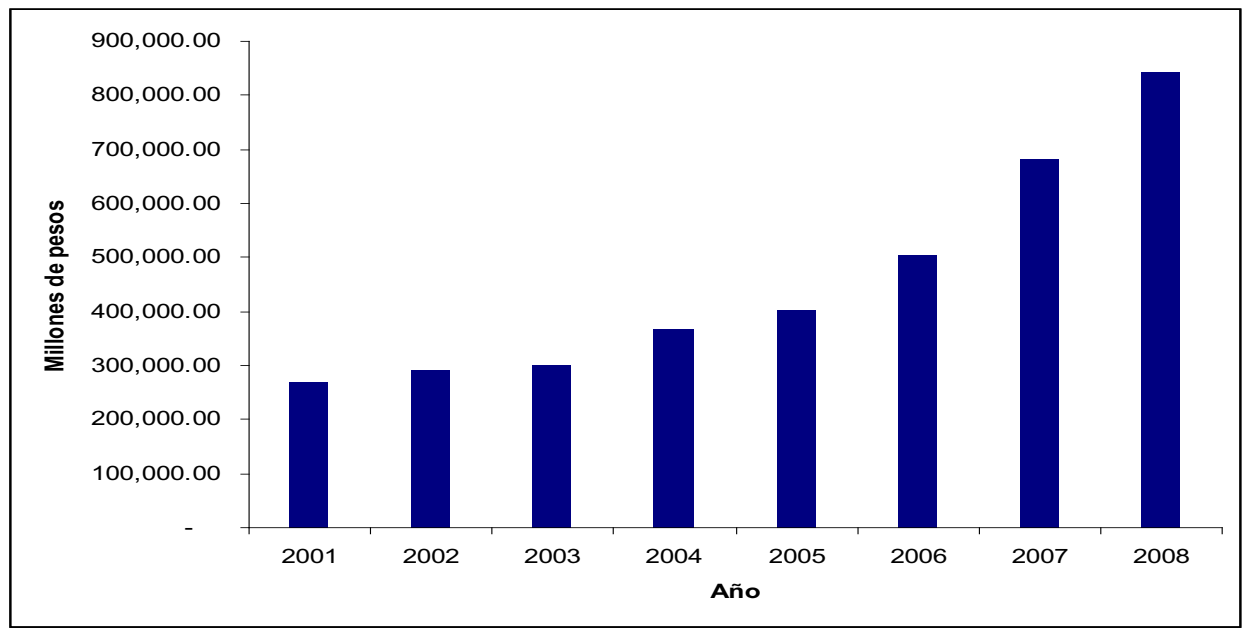

Fuente: Elaboración propia con datos de la balanza de intermediarios financieros publicada por el Banco Nacional de México (Banxico) a precios constantes de 2003.

${ }^{5}$ Fideicomiso creado por la Secretaría de Economía para financiar necesidades de capital de trabajo, o adquisición de activos fijos mediante un esquema que no requiere garantías. 
En el gráfico 1 se muestra el desempeño y evolución del crédito de la Banca Comercial, el cual en general presenta una tendencia a la alza, del año 2001 hasta el año 2008, como respuesta a los estímulos del mercado, así como a los programas del Gobierno Federal que promueven el apoyo a PYMES.

Para 2004 se constituyó el Sistema Nacional de financiamiento PYME, ${ }^{6}$ el cual tiene por objetivos:

- Promover y apoyar la constitución y fortalecimiento de fondos de garantía

- Desarrollar canales alternativos de financiamiento

- Diseñar más productos financieros para PYMES

- Mejorar condiciones de acceso al crédito

- Generar sujetos de crédito

En este contexto se desarrollaron dos programas: uno operado por Nacional Financiera (Fondo Paraguas); y otro por la Fundación para el Desarrollo Sostenible en México (FUNDES), Garantía PYME. Ambos comparten el objetivo de administrar el recurso aportado por el Fondo Pyme, y acordar con los bancos los términos de operación. Estos fondos fueron complementados por 26 estados de la República Mexicana con una aportación de aproximadamente 92.5 millones de pesos.

En la operación, del fondo paraguas participan por parte de la banca comercial Banorte, Bansí y al Banco del Bajío, Mifel. Fundes, por su parte, tiene participación de BBVA Bancomer, Santander Serfin y HSBC; este último cubre un porcentaje de la pérdida esperada de las instituciones, lo que permite que la banca evalúe el riesgo de otorgar financiamiento a estas empresas.

El programa con BBVA Bancomer se caracteriza por cubrir $4.5 \%$ de la pérdida esperada. Esta cobertura permite que el banco multiplique los recursos de la Secretaría de Economía en 20 a 1, para beneficiar a 2,600 empresas. A la fecha el programa con BBVA Bancomer (Tarjeta Negocios) ha beneficiado con crédito a 15,763 empresas, generando una derrama crediticia de más de 5 mil millones de pesos. Por su parte Santander-Serfin, a

${ }^{6}$ Fuente: Reportes de la Secretaría de Economía del Fondo PYME. 
través de su producto Crédito Ágil, impulsa el otorgamiento de créditos a 4,800 empresas, generando una derrama crediticia de 1,200 millones de pesos.

Asimismo, este programa de garantías cubre hasta $2.5 \%$ de la pérdida esperada y la multiplicación del recurso contemplada de 20 a 1. A la fecha esté programa ha apoyado a 15,519 empresas con una derrama de 4 mil millones de pesos. El programa de HSBC contempla una multiplicación del recurso de 20 a 1, y la generación de una derrama crediticia de 1,600 millones de pesos. A la fecha se han apoyado aproximadamente a 5 mil empresas, generando una derrama de 2 mil millones de pesos. Los productos que ofrecen estas instituciones tienen una tasa de interés competitiva y sin garantías hipotecaria.

El Programa de Garantía PYME opera a través del fideicomiso denominado "Fiso 8013-9" de Nacional Financiera, que es quien contragarantiza créditos desde $\$ 30,000.00$ (treinta mil pesos 00/100) hasta un máximo de 2.7 millones de pesos, equivalente a 900 mil UDIS (novecientas mil unidades de inversión), otorgados por la banca comercial, sin garantía hipotecaria, con tasa de interés competitiva (conocida y topada). Las garantías del fondo Paraguas cubren hasta $75 \%$ en créditos Pyme a empresas en operación y hasta $80 \%$ para empresas de reciente creación. Los recursos aportados en 2004 tienen como meta generar una derrama crediticia de 600 MDP, multiplicando el recurso de la Secretaría de Economía en 20 a 1.

En este esquema las garantías son pagadas en el momento del reclamo, tras la verificación del cumplimiento de los requisitos de crédito garantizado. Posteriormente y con periodicidad semestral, las entidades financieras deben informar a Nafin sobre los avances en los procesos de cobro.

El Programa de Pérdida Esperada para Empresas Exportadoras tiene como fin impulsar el otorgamiento de créditos en condiciones accesibles, oportunas y competitivas a las PYMES exportadoras. Este programa opera con diferentes instituciones financieras. Bancomext solicitó apoyo para instrumentar un programa de crédito a las PYMES a través de la banca múltiple, en donde requiere la cobertura de la pérdida esperada.

Los créditos que otorgan los bancos comerciales participantes en el programa tienen una tasa de TIIE (tasa de interés interbancaria de equilibrio) más 10 puntos porcentuales $(\mathrm{MN})$ o tasa Libor (London Interbank 
Offer Rate) más 10 puntos porcentuales (USD). El monto máximo y mínimo de los créditos que se apoyan con el proyecto es de USD 10,000.00 (diez mil dólares) y USD 200,000.00 (doscientos mil dólares) respectivamente por destino, sin garantías reales y con un plazo de 36 meses para capital de trabajo, y hasta 5 años para activos fijos, pudiendo incluir un periodo de gracia.

La Secretaría de Economía, en coordinación con Nacional Financiera, puso a disposición de los intermediarios financieros los recursos fiscales provistos por la Secretaría y administrados por Nacional Financiera, S.N.C. a través de un proceso de Selección de productos Pyme.

El objetivo de dicho proceso es evaluar los productos crediticios que propongan los bancos, para ser garantizados bajo la cobertura de la pérdida esperada a través de la Garantía Pyme. Se consideraron como criterios básicos el múltiplo y la tasa de interés, y de acuerdo con los requerimientos se determinó el monto de recursos que serían reservados para la cartera de los bancos cuyos productos hayan sido seleccionados. El objeto era beneficiar con crédito a más de 10,00o PYMES, cubriendo hasta 4\% de los incumplimientos de la cartera crediticia aproximada de cinco mil millones de pesos.

Durante 2005 se llevaron a cabo dos procesos de selección. En el primero participaron Banamex (crédito revolvente), Scotia Bank Inverlat (Scotia Pyme), Santander Serfin (crédito Pyme y Crédito Ágil) y Bancomer (tarjeta Negocios). En el segundo Proceso de Selección participaron HSBC (crédito a negocios y crédito automotriz), Santander Serfin (Crédito Pyme Rec. Propios y Crédito ágil) y Banco del Bajío (crédito MIPyme y crédito Pyme).

Los productos presentados en los dos procesos de selección son sin garantía hipotecaria, con una tasa máxima de TIIE+12 puntos, con una multiplicación promedio de recursos de 42 a 1; para generar una derrama aproximada de 13 mil millones de pesos.

De acuerdo con información proporcionada por la Dirección de Promoción Empresarial de la Subsecretaría para la Pequeña y Mediana Empresa, los resultados del programa de garantías han sido los siguientes. (Ver cuadro 1). 
Cuadro 1.- PYMES Apoyadas y Crédito Garantizado

\begin{tabular}{|l|c|c|c|}
\hline Año & $\begin{array}{c}\text { Pymes } \\
\text { (número de empresas ) }\end{array}$ & $\begin{array}{c}\text { Créditos } \\
\text { (número de créditos) }\end{array}$ & $\begin{array}{c}\text { Derrama } \\
\text { (pesos) }\end{array}$ \\
\hline 2002 & $3,012.00$ & $3,330.00$ & $162,658,003.20$ \\
\hline 2003 & $12,815.00$ & $14,591.00$ & $2,081,991,170.82$ \\
\hline 2004 & $23,692.00$ & $25,115.00$ & $6,721,428,430.57$ \\
\hline 2005 & $46,156.00$ & $71,932.00$ & $15,027,484,333.90$ \\
\hline 2006 & $72,200.00$ & $142,501.00$ & $23,900,980,926.06$ \\
\hline 2007 & $81,138.00$ & $122,212.00$ & $21,792,472,496.61$ \\
\hline $\begin{array}{c}2008 \\
\text { (Hasta } \\
\text { marzo) }\end{array}$ & $4,269.00$ & $5,182.00$ & $4,581,471,281.41$ \\
\hline
\end{tabular}

Fuente: Subsecretaría para la Pequeña y Mediana Empresa, Secretaría de Economía.

Como se podrá observar, se ha mantenido un crecimiento de los apoyos otorgados en los programas año con año. Es importante señalar que en el año 2004 la derrama generada tuvo un incremento mayor al registrado en el número de empresas apoyadas.

Por otra parte, el mayor número de empresas apoyadas pertenece al comercio. Con los nuevos programas que se orientan al financiamiento de maquinaria y equipo se incrementarán los apoyos al sector industrial. (Ver cuadro 2.)

Cuadro 2.- PYMES Apoyadas y Derrama Económica por Sector

\begin{tabular}{|l|c|c|}
\hline SECTOR & $\begin{array}{c}\text { PYMES } \\
\text { (número de empresas ) }\end{array}$ & $\begin{array}{c}\text { DERRAMA } \\
\text { (pesos) }\end{array}$ \\
\hline COMERCIO & $45,958.00$ & $10,502,968,511.00$ \\
\hline INDUSTRIA & $64,648.00$ & $12,061,509,958.00$ \\
\hline SERVICIOS & $45,915.00$ & $10,395,564,112.00$ \\
\hline TOTAL & $156,521.00$ & $32,960,042,581.00$ \\
\hline
\end{tabular}

Fuente: Subsecretaría para la Pequeña y Mediana Empresa, Secretaría de Economía.

La distribución de los apoyos del periodo (2002-2008) por tamaño, muestra una tendencia al equilibrio. En los primeros tres años (20022008) la pequeña y la mediana empresa concentraba el mayor número de 
apoyos, en los últimos años (2004-2008) se concentraron en la microempresa. La micro presenta una importante participación en los apoyos a partir de 2004, ya que en años anteriores el porcentaje de apoyos se concentraba en la mediana empresa. Para 2004 un tercio de las empresas apoyadas ya son micro (las empresas apoyadas están repartidas proporcionalmente). Así, al cierre global la micro es la más favorecida, seguida por la pequeña y por último la mediana. (Ver cuadro 3.)

Cuadro 3.- Total de apoyos por Segmento

\begin{tabular}{|l|c|r|}
\hline SECTOR & $\begin{array}{c}\text { PYMES } \\
\text { (número de empresas) }\end{array}$ & $\begin{array}{c}\text { DERRAMA } \\
\text { (pesos) }\end{array}$ \\
\hline MICRO & $87,419.00$ & $15,284,254,009.00$ \\
\hline PEQUEÑA & $42,545.00$ & $9,152,991,057.00$ \\
\hline MEDIANA & $26,557.00$ & $8,522,797,515.00$ \\
\hline TOTAL & $156,521.00$ & $32,960,042,581.00$ \\
\hline
\end{tabular}

Fuente: Subsecretaría para la Pequeña y Mediana Empresa, Secretaría de Economía.

\section{V.- Evidencia Empírica}

Para determinar el impacto del Sistema Nacional de Garantías en el crecimiento económico en México, primero se analiza el modelo planteado por Craig, Jackson y Thomson (2005), quienes a través del método de mínimos cuadrados ordinarios miden el impacto que tiene el sistema de garantías en el crecimiento económico. Dicho modelo básico resulta:

PICAP $_{t}=\alpha+\alpha_{1} P I C A P_{t-1}+\alpha_{2} S B A D E P_{t-1}+\alpha_{3} E_{1 M P R_{t}}+\alpha_{4} N B E R_{t}+\alpha_{5} H E R F_{t}+\alpha_{6} S B A G_{t-1}$ $+\alpha_{7} S B A 7 A_{t-1}+\alpha_{8} S B A M_{t-1}+\alpha_{9} M D U M_{t-1}+\varepsilon_{t}$

En donde:

PICAP $_{t}=$ Representa el Producto Interno Bruto Per Cápita en el periodo t. PICAP $_{t-1}=$ Representa el Producto Interno Bruto Per Cápita en el periodo t-1.

$S B A D E P_{t-1 t}=$ El monto total de garantías otorgadas a los bancos en el periodo t-1. 
$E M P R_{t}=$ Tasa de desempleo mensual en el periodo t.

$N B E R_{t}=$ Variable dummy que determina si la economía está en crecimiento o no ( $1=$ en recesión, $\mathrm{O}=$ Otras situación)

$C V E_{t}=$ Representa el monto de la cartera vencida de créditos comerciales otorgados por la banca en el periodo t.

$H E R F_{t}=$ El monto de los depósitos efectuados en la banca comercial en el periodo t.

$S B A G_{t-1}=$ Monto de los créditos otorgados a través del sistema de garantías en el periodo t.

$S B A M_{t-1}=$ El importe de los créditos otorgados por la banca comercial cuyo destino es el consumo en el periodo $\mathrm{t}-1$.

$M D U M_{t-1}=$ El monto total de créditos otorgados a las empresas por parte de la banca comercial.

$\varepsilon_{t}=$ Representa un componente aleatorio (los residuos) que recoge todo lo que las variables independientes no son capaces de explicar.

A partir de este modelo y considerando las aportaciones realizadas por Arellano y Bond (1991) se plantea el modelo:

PIBPC $_{t}=\alpha+\beta_{1}$ PIBPC $_{t-1}+\beta_{2}$ GARANTIA $_{t}+\beta_{3}$ CREDPYME $_{t}+\beta_{4}$ CRECONS $_{t}+\beta_{5}$ DESP $_{t}+\beta_{6} R E S_{t}$ $+\beta_{7} P Y M E_{t}+\varepsilon_{t}$

PIBPC $_{t}=$ Representa el Producto Interno Bruto Per Cápita en el periodo t. PIBPC $_{t-1}=$ Representa el Producto Interno Bruto Per Cápita en el periodo t-1.

GARANTIA $_{t}=$ Monto de las garantías otorgadas a la banca comercial en el periodo t. 
CREDPYME $E_{t}=$ Cantidad total de los créditos otorgados a las PYMES a través del Sistema Nacional de Garantías.

CRECONS $S_{t}=$ Monto de los créditos otorgados al consumo.

$D E S P=$ Monto total de los créditos otorgados al sector empresarial.

$R E S_{t}=$ Variable dummy que determina si la economía está en crecimiento o no ( $1=$ en recesión, $\mathrm{O}=$ Otras situación).

$P Y M E_{t}=$ Número de PYMES que recibieron financiamiento a través del programa de garantías.

Cabe mencionar que este modelo cumple con los supuestos de linealidad, independencia, normalidad, homocedasticidad y no-colinealidad.

Para llevar a cabo la prueba se utilizan las series históricas mensuales, correspondientes al periodo comprendido del 1 de enero de 2002 al 30 de marzo de 2008, de los préstamos otorgados por la banca comercial (tanto al consumo como al sector empresarial), los montos destinados a las garantías liquidas, los préstamos otorgados a través del Sistema Nacional de Garantías y el número de PYMES beneficiadas. Las fuentes de información consultadas son las bases de datos del Banco de México (Banxico); el Instituto Nacional de Estadística, Geografía e Informática (INEGI); la Secretaría de Economía (SE) y la Asociación de Bancos de México.

La serie correspondiente al Producto Interno Bruto per Cápita se estima con base en la información proporcionada por el INEGI y Banxico; además, se integra mensualmente a precios corrientes. Es importante mencionar que el INEGI estima el PIB en forma trimestral, por lo que pudiera representar un problema debido a que movimientos que pudieran ser significativos en un mes se ven suavizados por el uso de esta serie.

\section{Resultados}

$\mathrm{Al}$ realizar la regresión:

PIBPC $_{t}=\alpha+\beta_{1}$ PIBPC $_{t-1}+\beta_{2}$ GARANTIA $_{t}+\beta_{3}$ CREDPYME $_{t}+\beta_{4}$ CRECONS $_{t}+\beta_{5}$ DESP $_{t}+\beta_{6}$ RES $_{t}$ $+\beta_{7} P Y M E_{t}+\varepsilon_{t}$ 
Tabla 1.- Resumen del modelo

\begin{tabular}{|c|c|c|c|c|c|}
\hline Model & R & R Square & Adjusted R Square & Std. Error of the Estimate & Durbin-Watson \\
\hline 1 & $.981 \mathrm{a}$ & .962 & $.95^{8}$ & 8012.62523 & 1.195 \\
\hline
\end{tabular}

b. Dependent Variable: PIBPC

La tabla 1 muestra que todas las variables independientes incluidas en el análisis explican un $98 \%$ de la varianza de la variable dependiente, pues R 2 corregida $=.958$.

Por otra parte, el estadístico F contrasta la hipótesis nula de que el valor poblacional de $\mathrm{R}$ es cero $\mathrm{y}$, por tanto, nos permite decidir si existe relación lineal significativa entre la variable dependiente y el conjunto de variables independientes tomadas juntas. El valor del nivel crítico Sig. = o,ooo indica que sí existe relación lineal significativa. (Ver tabla 2).

\begin{tabular}{|c|c|c|c|c|c|c|}
\hline \multicolumn{7}{|c|}{$\begin{array}{c}\text { Tabla 2.- ANOVA } \\
\text { ANOVA }^{\mathrm{b}}\end{array}$} \\
\hline \multicolumn{2}{|c|}{ Model } & Sum of Squares & $\mathrm{df}$ & Mean Square & $\mathrm{F}$ & Sig. \\
\hline \multirow[t]{3}{*}{1} & Regression & $1.072 \mathrm{E} 11$ & 7 & $1.532 \mathrm{E} 10$ & 238.585 & $.000 a$ \\
\hline & Residual & $4.237 \mathrm{E} 9$ & 66 & $6.420 \mathrm{E} 7$ & & \\
\hline & Total & 1.115E11 & 73 & & & \\
\hline
\end{tabular}

a. Predictors: (Constant), PYME, RECS, DESP, CREDCONS, RPIBPC, GARANTÍA, CREDPYME

b. Dependent Variable: PIBPC

Por lo que respecta a los valores de los coeficientes de cada una de las variables y al nivel de significancia de cada una de ellas, se observa que coeficiente correspondiente a las garantías es positivo y significante. Esto coincide con los resultados de Craig, Jackson y Thomson (2005) quienes identificaron una relación directa entre la derrama económica derivada de las garantías y el crecimiento económico (medido a través del PIB Per Cápita). (Ver tabla 3). 
Tabla 3.- Coeficientes

\begin{tabular}{|c|c|c|c|c|c|c|}
\hline \multirow{2}{*}{\multicolumn{2}{|c|}{ Model }} & \multicolumn{2}{|c|}{ Unstandardized Coefficients } & \multirow{2}{*}{$\begin{array}{c}\text { Standardized } \\
\text { Coefficients } \\
\text { Beta }\end{array}$} & \multirow[b]{2}{*}{$\mathrm{t}$} & \multirow[b]{2}{*}{ Sig. } \\
\hline & & B & Std. Error & & & \\
\hline \multirow[t]{8}{*}{1} & (Constant) & 241244.544 & 40385.552 & & 5.974 & .000 \\
\hline & RPIBPC & .527 & .069 & .523 & 7.646 & .000 \\
\hline & GARANTIA & 26.370 & 7.176 & .516 & 3.675 & .000 \\
\hline & CREDPYME & 4.133 & 1.558 & .471 & 2.653 & .003 \\
\hline & CREDCONS & -.004 & .005 & -.028 & -.723 & .472 \\
\hline & DESP & 6977.513 & 3175.326 & .082 & 2.197 & .032 \\
\hline & RECS & -22437.853 & 2358.752 & -.287 & -9.513 & .000 \\
\hline & PYME & 5.814 & 2.244 & .361 & 2.591 & .012 \\
\hline
\end{tabular}

a. Dependent Variable: PIBPC

En cuanto a los créditos otorgados a las PYMES, éstos presentan un coeficiente positivo y significante, lo que muestra una relación directa entre el crecimiento económico y el financiamiento a empresas con el desempeño económico.

\section{VI.- Conclusiones}

Los sistemas de garantía para préstamos a PYMES son un instrumento de intervención del gobierno en los mercados crediticios y su objetivo es el de promover el desarrollo de este tipo de empresas. La implementación de este tipo de programas se justifica debido a que en la economía se presentan fallos en el mercado como el racionamiento crediticio. Para el caso de México como consecuencia de la crisis de 1995, la dificultad para las PYMES de obtener créditos bancarios se profundizó propiciando un racionamiento crediticio hasta nuestros días. La disminución de la oferta crediticia se sustenta en que los bancos consideran a las pequeñas empresas más riesgosas que las empresas grandes, ya que las primeras tienen menos historia, menos capital propio, menos experiencia y están compitiendo en mercados saturados. 
Al no contar con financiamiento las PYMES no logran desarrollarse, lo que afecta el crecimiento económico del país; esto debido a que las PYMES representan $99.8 \%$ del total de empresas establecidas, contribuyen con $52 \%$ del PIB y generan $52 \%$ del total de empleos. ${ }^{7}$ Ante esta situación, la Secretaría de Economía implementó en 2002 el Sistema Nacional de Garantías cuyo objetivo principal es ayudar a las PYMES a acceder a financiamiento y con ello generar las condiciones necesarias para el crecimiento en la economía. De acuerdo con los resultados obtenidos en esta investigación, se puede determinar que hasta el momento dicho sistema está cumpliendo con su finalidad, aunque esto debe interpretarse con cierta mesura debido a varias razones. Primero, los préstamos concedidos a las empresas están determinados por la oferta, esto debido a que los contratos no han incentivado la expansión de la cartera de créditos a las PYMES que no tenían acceso al sistema financiero formal. Condiciones como el nivel mínimo de ventas anuales, información financiera de los últimos dos años, contar con obligado solidario, constituyen limitaciones para cumplir con el objetivo.

Por otro lado, la expansión registrada en el número de PYMES beneficiadas en gran medida puede estar originado por el crecimiento natural y reorientación del mercado objetivo, pero no por el efecto de la garantía; es decir, si bien el esquema de garantías ha permitido a los bancos cubrirse mejor contra el riesgo de incumplimiento, no explica, del todo, el crecimiento en los créditos otorgados. Debido a la creciente competencia entre los bancos y otras instituciones financieras, ha obligado a los primeros a incursionar a nuevos mercados.

\footnotetext{
${ }^{7}$ Fuente: Libro blanco Fondo PYME 2006 de la Secretaría de Economía.
} 


\section{Bibliografía}

Alvarado, J. (2002) "Los Fondos de Garantía para el Acceso al Crédito en el Perú: alcances y limitaciones”, Informe final presentado al CIESS/Red de Microcrédito, Género y Pobreza, Centro Peruano de Estudios Sociales, octubre.

Belloc, B y Freixas, L. (1988). Equilibrium and Rationing in the Credit Market: A Sequential Equilibrium Approach, Cahier du GREMAO, Université de Toulouse.

Craig, B., Jackson, W. y Thomson (2005). "On SBA Guaranteed Lending and Economic Growth", Government-University Partnerships to Enhance Economic Development Trhough Enterpreneurship, Edwar Elgar Publising.

De Meza, D. y Webb, C. (2005)."Risk, Adverse selection and Capital Market Faiule”, Economic Journal Núm. 100.

Gale, W. (1990). "Federal Lending and the Market for Credt", Journal of Public Economy, Vol. 42, Núm. 3, julio.

Gale, W. (1990). "Collateral, rationing and Government Intervention in Credit Markets”, en R. G. Hubbard (Edit.), Asmmetric Information, Corporate Finance and Investment, University of Chicago Press, pp. 43-61.

Gómez E. y Reyes R. (2002). "El racionamiento del crédito y las crisis financieras”, Revista de Economía Institucional, Vol.4, Núm. 7, segundo semestre.

Gudger, M. (1998). Credit Garantees: An Assesssment of the State of Knowledge and News Avenues of Research. FAO.

Holdel P. (1997). "Collateral Without Consecuence: Some Causes and Effects On Financial Unerdevelopment in Latin America”, en Juan José Listerri, The Financier, Analyses of Capital and Money Market Transaction; Banco Interamericano de Desarrollo 1997. 
Hubbard, R. (1986). "Capital Markets Imperfections and Investment”, Journal of Economics Literature, Núm. 36.

Jaffe, D y Modigliani, F. (1969). A Theory and Tets of Credit Rationing, American Economic Review, Núm. 59.

Jaffe, D y Stiglitz, J. “Credit Rationing”. En B.M. Friedman and F.H. Hahn Editores, The Handbook of Monetary Economics, Volumen II, North-Holland, Amsterdam, 1990.

Lacker M. (1994).“Does Adverse Seletion Justify Government Intervention in Loan Markets?”, Economic Quaterly, Volumen 80, invierno .

Li, W. (1998). "Government Loan, Guarantee, and Grant Programs: An Evaluation”, Economic Quaterly, Volumen 84, otoño.

Parker S. (2002). "Do Banks Ration Credit to New Enterprises? And Should Governments Intervene?”, Scottish Journal of Political Economy, Vol. 49, Núm. 2, mayo.

Parker, S. (2003). "Asymmetric Information, Ocuppational Choise and Government Policy”, Ecomyc Journal, Vol. 11, Núm. 3. otoño.

Secretaría de Economía (2008). "Reporte del Desarrollo del Sistema Nacional de Garantías 2002-2008”.

Smith B. y Stutzer M. (1989). "Credit Rationing and Government Loan Programs: A Welfare Analysis”, AREUEA Journal, Volumen 17, Núm. 2.

Stearns, C. (1993). Fondos de Garantía y sus Posibilidades para la Microempresa, Cambridge M.A.: Acción Internacional, agosto 1993.

Stiglitz, J. y Weiss A. (1981). "Credit Rationing in Markets with Imperfect Information”, The American Economic Review, Volumen 71, Núm. 3, junio.

Mankiw, N. (1983). "The Allacation of Credit and Finance Collapse”, Quaterly Journal of Economics, Num. 101. 